\title{
Grand canyon des gorges du Verdon
}

Jean Gagnepain

URL : http://journals.openedition.org/adlfi/6737

ISSN : 2114-0502

Éditeur

Ministère de la culture

Référence électronique

Jean Gagnepain, "Grand canyon des gorges du Verdon », ADLFI. Archéologie de la France - Informations [En ligne], Provence-Alpes-Côte d'Azur, mis en ligne le 01 mars 2007, consulté le 20 avril 2019. URL http://journals.openedition.org/adlfi/6737

Ce document a été généré automatiquement le 20 avril 2019

(c) Ministère de la Culture et de la Communication, CNRS 


\title{
Grand canyon des gorges du Verdon
}

\author{
Jean Gagnepain
}

Identifiant de l'opération archéologique : 8316

Date de l'opération : 2007 (PI)

1 En 2007, le Musée de Préhistoire des Gorges du Verdon a initié un programme de prospection-inventaire sur le territoire du Grand Canyon des Gorges du Verdon. Ce programme, planifié sur plusieurs décennies, intègre des opérations de fouille ou de sondage plus ponctuelles, comme en 2006 au dolmen des Pierres Blanches à Castellane (voir BSR PACA 2006 : 24-26) ou à Moustiers-Sainte-Marie en 2007.

2 Les communes concernées par la prospection sont Moustiers-Sainte-Marie, La Palud-surVerdon, Rougon et Castellane dans les Alpes-de-Haute-Provence et Le Bourguet, Trigance, Comps-sur-Artuby et Aiguines dans le Var. La première phase du programme concerne les communes de la rive droite (Alpes-de-Haute-Provence), avec un accent particulier mis dans un premier temps sur Moustiers-Sainte-Marie.

3 Sur un plan méthodologique, trois approches seront développées :

4 La recherche bibliographique a été entamée et se poursuivra dans les années futures; de nombreux témoignages ont été consignés et répertoriés, avec plusieurs visites des sites à Castellane, La Palud-sur-Verdon ou Moustiers-Sainte-Marie. Certains témoignages et déplacements sur site ont été suivis d'opérations de terrain en sauvetage urgent : site de Font Collomb à Moustiers fin 2007 (structures et mobilier néolithiques et modernes) et sépulture gallo-romaine de la Campagne de Serre prévue en janvier 2008.

5 La prospection pédestre n'a concerné en 2007 que la commune de Moustiers-SainteMarie. Elle a débuté en avril par la zone de plateau (limite orientale de la formation de Valensole) et les champs de lavande et s'est poursuivie à l'automne par les rives du lac de Sainte-Croix, au niveau particulièrement bas (Fig. $\mathrm{n}^{\circ} 1$ : Prospection pédestre sur les rives du lac de Sainte-Croix). Outre du matériel épars, quinze sites ont été mis en évidence, dont quatorze à priori inédits (quatorze fiches de découverte, une fiche de révision pour l'établissement gallo-romain de Saint-Saturnin). 
Différentes phases chronologiques sont représentées : Préhistoire (quatre sites), Antiquité (trois sites), époque moderne (un site), époque moderne/contemporaine (cinq sites), époque contemporaine (deux sites).

7 La plupart du temps, la présence de structures (surtout des structures en élévation, mais aussi des structures en creux) est à l'origine du repérage des sites (onze fiches). Il s'agit majoritairement de vestiges attribués, d'après le mobilier associé (céramique vernissée à décor d'engobe rapporté), à l'époque moderne/contemporaine: cinq habitats et un oratoire inédit présentant un bénitier en façade (zone de Saint-Pons) ainsi que, plus récentes, deux carrières d'exploitation des galets du poudingue situées en bordure sudest du plateau de Valensole. On recense par ailleurs deux sites d'âge gallo-romain (dolium, amphore, céramiques sigillée et claire récente), d'organisation plus ou moins complexe (Saint-Saturnin et les Pradelles), et un site néolithique caractérisé par des fosses contenant des pans de céramique néolithique moyen/final (pointe de Font Collomb).

Des concentrations de mobilier lithique ou céramique ont également été repérées (quatre fiches). Elles permettent d'ajouter à l'inventaire des découvertes un site gallo-romain (Godemar 1) et trois occupations d'âge préhistorique: deux stations paléolithiques - Godemar 2 et Cap de la Borne (?) - et une nappe de vestiges céramiques néolithiques (plage de Saint-Saturnin).Trois secteurs ont particulièrement livré des concentrations de vestiges :

En conclusion, nous pouvons souligner les résultats très positifs de cette première année de prospection, avec une forte anthropisation diachronique des secteurs inventoriés et une grande variété de sites et de typologies de structures et de mobilier.

Les relations très positives avec les entités administratives territoriales et avec la population locale laissent augurer d'une relation fructueuse avec l'équipe de recherche et donc de résultats conséquents.

\section{GAGNEPAIN Jean}

Cette opération a été conduite avec la collaboration de Yann Dedonder, Jean-François Devos, Isabelle Dubset, Caroline Luzi, Vincent Meyer, Laurence Mombel et Jean-Luc Ramu. Nous tenons particulièrement à remercier les services de l'État (SRA DRAC-PACA), le Conseil général des Alpes-de-Haute-Provence, la commune de Moustiers-Sainte-Marie, le Parc naturel régional du Verdon ainsi que Sébastien Molinatti.

\section{ANNEXES}


Fig. $n^{\circ} 1$ : Prospection pédestre sur les rives du lac de Sainte-Croix

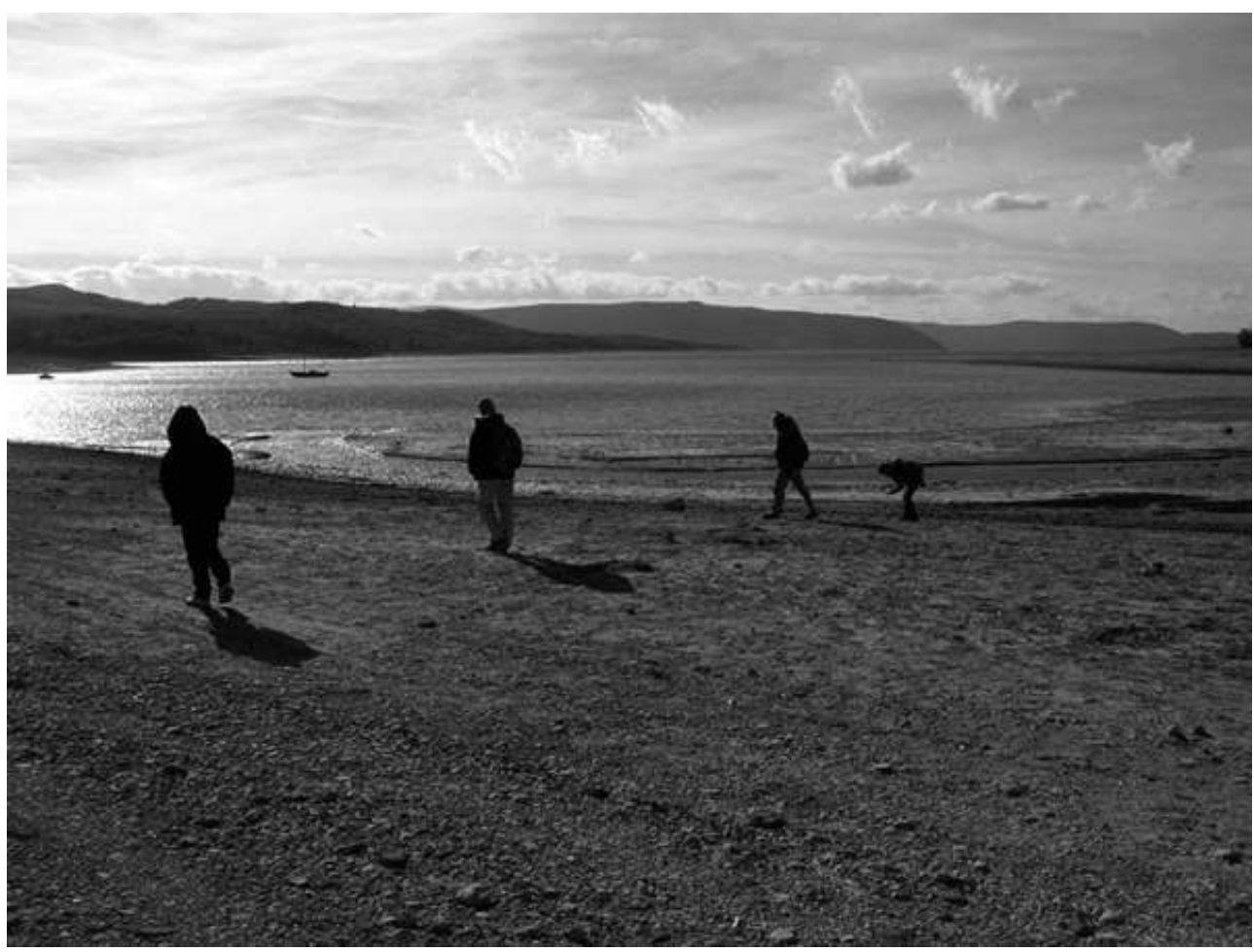

Auteur(s) : Gagnepain, Jean. Crédits : ADLFI (2007)

\section{INDEX}

Index chronologique : Empire romain, ép. contemporaine, Paléolithique, Préhistoire, Temps Modernes

operation Prospection inventaire (PI)

Index géographique : Provence-Alpes-Côte d'Azur, Alpes-de-Haute-Provence (04), Var (83), Moustiers-Sainte-Marie, Rougon, Castellane, Trigance

\section{AUTEURS}

\section{JEAN GAGNEPAIN}

COL 